EDITORIAL

\title{
Clinical applications of tissue Doppler imaging: a promise fulfilled
}

\section{T H Marwick}

Heart 2003;89:1377-1378

The clinical applications of tissue Doppler imaging in the assessment of left ventricular diastolic function have moved from the experimental lab to clinical practice

T issue Doppler imaging, which uses Doppler to quantify the velocity of tissue rather than blood, has been available as a clinical tool for over a decade. A previous commentary in Heart has emphasised the technical aspects, ${ }^{1}$ so the purpose of this brief review is to assess whether this technology really has contributed to clinical evaluation, and other areas where additional development is needed.

One of the limitations to the uptake of this modality has been the plethora of technical approaches to measuring tissue Doppler parameters. The simplest approach is to measure regional velocity using pulsed wave Doppler, which has the attraction of very high temporal resolution, but the disadvantage of fixing the localisation of the sample volume at the time of study acquisition. As with all Doppler, the direction of motion should be as close as possible to the scan line direction, and careful attention needs to be paid to gain settings, because excessive gain leads to spectral broadening and overestimation of velocities. The alternative is the acquisition of colour tissue Doppler, which has the attraction of permitting extensive off-line analysis, although high frame rate is critical in order to maximise the information gathered in the Doppler profile. The simplest and most robust parameters are peak systolic and diastolic tissue velocities, which have high levels of concordance between observers. Although temporal parameters are very sensitive indices to myocardial dysfunction, the measurement of these parameters shows more variability. Myocardial velocity gradients and strain rate are derived from the comparison of adjacent velocities and therefore minimise the influence of tethering and translation. However, as these variables are derived from multiple measured parameters, signal noise is a bigger problem than with simple tissue velocity.

Correspondence to: Professor Thomas Marwick, University of Queensland Department of Medicine, Princess Alexandra Hospital, Ipswich Road, Brisbane, Qld 4012, Australia; tmarwick@ soms.uq.edu.au

\section{ASSESSING LV DIASTOLIC FUNCTION}

The greatest clinical contribution of tissue Doppler to date has almost certainly been in the assessment of left ventricular diastolic function. Although the analysis of transmitral blood flow has improved the feasibility of diastolic function assessment, the use of this parameter in isolation is confusing because it behaves in a non-linear fashion as diastolic function deteriorates. $^{2}$ A number of techniques have been developed to differentiate between normal and pseudo-normal left ventricular filling patterns, but arguably the simplest approach is the measurement of annular tissue velocity. While this parameter is not independent of large changes of preload in a normal ventricle (for example, during dialysis) it appears to be much less influenced by preload in an impaired ventricle, and does not pseudo-normalise in the same way as the transmitral flow. Reduced annular velocity $(\mathrm{Ea}<8)$ in association with apparently normal transmitral flow is therefore a marker of pseudo-normal filling. Unfortunately, the normal ranges in the septal and lateral walls are different, and the normal range falls with age.

A second and related application of tissue Doppler to routine clinical practice is the assessment of left ventricular filling pressure. ${ }^{3}$ Again, a number of techniques may be used for this purpose, including comparison of transmitral filling with flow propagation velocity and the duration of the pulmonary vein atrial reversal. Nonetheless, a very simple approach is to obtain a ratio of the $\mathrm{E}$ wave (which is dependent on left atrial pressure) and the Ea velocity by tissue Doppler (which is relatively independent of left atrial pressure). If the resulting number is $<8$, it is likely that left ventricular filling pressure is normal; conversely, if the ratio is $>15$ it is very unlikely that filling pressure is less than $15 \mathrm{~mm} \mathrm{Hg}$. A ratio of $8-15$ is more ambiguous, and can be resolved by assessment of pulmonary venous atrial reversal duration or responses to the Valsalva manoeuvre.

\section{SYSTOLIC CONTRACTION}

The temporal evaluation of left ventricular systolic contraction promises to become the third important clinical application of tissue Doppler. As the ventricle enlarges in heart failure, systolic function becomes progressively less synchronous, which itself contributes to reduced left ventricular efficiency. The recent development of resynchronisation therapy in heart failure has resulted in improvement of exercise capacity and quality of life, but approximately $20 \%$ of patients fail to respond to treatment. Although electrocardiographic QRS duration is mostly widely used in the selection of patients for this expensive intervention, it appears that the use of tissue Doppler to measure the delay between each of the walls is an efficient means of both selection of patients ${ }^{5}$ and also guidance for the location of biventricular pacing leads. ${ }^{6}$ 
Quantification of global and regional left ventricular systolic function is a promising "pipeline" indication for tissue Doppler, that is on the cusp of clinical application. The most important practical constraints relate to the normal gradation of tissue velocity from base to the apex and from one side of the heart to the other, requiring the definition of normal ranges, which are also influenced by age. ${ }^{7}$ While this level of heterogeneity is reduced at peak dobutamine stress, leading to a successful approach for quantification of dobutamine echo using tissue Doppler, ${ }^{8}$ the resting application of this technique remains problematic. Because strain rate imaging avoids the limitations of tethering and translation, it seems likely that this will be the optimal technique for regional functional assessment, although alignment of the direction of the contraction with the Doppler beam remains a critical issue and signal noise is an ongoing concern. Nonetheless, the systolic velocity or displacement of the base of the heart, particularly when averaged across multiple walls, correlates well with ejection fraction, and may be an effective means of assessing the cardiac contractile response to exercise.

\section{SUBCLINICAL LV DYSFUNCTION}

The final (and perhaps the most exciting) application of tissue Doppler techniques is in the detection of subclinical left ventricular dysfunction. Myocardial velocity gradients have been shown to be abnormal in patients with subclinical left ventricular dysfunction, and the degree of abnormality in hereditary diseases such as Friedrich's ataxia appears to correlate with the degree of genetic abnormality. ${ }^{9}$ Moreover, abnormal tissue velocities can be used to differentiate between pathologic left ventricular hypertrophy and athletic left ventricular hypertrophy, ${ }^{10}$ and a particularly exciting application has been in the detection of patients with undeclared hypertrophic cardiomyopathy who have a family history of the disease and an abnormal genotype. ${ }^{11}$ For similar reasons the tissue Doppler approaches have been effective in the identification of transplant rejection ${ }^{12}$ and the detection of preclinical left ventricular impairment in the presence of regurgitant valve lesions. ${ }^{13}$ In all of these situations, the efficacy of tissue Doppler is very likely based upon its correlation with the degree of ventricular fibrosis, and the technique represents a more clinically feasible approach than previous attempts to identify subclinical heart disease with, for example, myocardial backscatter assessment.

The clinical applications of tissue Doppler in the assessment of left ventricular diastolic function, loading conditions, and synchronisation have moved from the experimental lab to clinical practice. Further advances, particularly in strain and strain rate imaging, will establish the use of this technique to quantify left ventricular systolic function.

\section{REFERENCES}

1 Sutherland GR, Lange A, Palka $P$, et al. Does Doppler myocardial imaging give new insights or simply old information revisited? Heart 1996;76:197-9.

2 Garcia MJ, Thomas JD, Klein AL. New Doppler echocardiographic applications for the study of diastolic function. J Am Coll Cardiol 1998;32:865-75

3 Ommen SR, Nishimura RA, Appleton CP, et al. Clinical utility of Doppler echocardiography and tissue Doppler imaging in the estimation of left ventricular filling pressures: a comparative simultaneous Dopplercatheterization study. Circulation 2000;102:1788-94.

4 Nagueh SF, Mikati I, Kopelen HA, et al. Doppler estimation of left ventricular filling pressure in sinus tachycardia. A new application of tissue Doppler imaging. Circulation 1998;98:1644-50.

5 Sogaard P, Egeblad H, Kim WY, et al. Tissue Doppler imaging predicts improved systolic performance and reversed left ventricular remodeling during long-term cardiac resynchronization therapy. J Am Coll Cardiol 2002:40:723-30.

6 Ansalone G, Giannantoni P, Ricci R, et al. Doppler myocardial imaging to evaluate the effectiveness of pacing sites in patients receiving biventricular pacing. J Am Coll Cardiol 2002;39:489-99.

7 Hatle L, Sutherland GR. Regional myocardial function-a new approach. Eur Heart J 2000;21:1337-57.

8 Cain P, Baglin T, Case C, et al. Application of tissue Doppler to interpretation of dobutamine echocardiography: comparison with quantitative coronary angiography. Am J Cardiol 2001;87:525-31.

9 Dutka DP, Donnelly JE, Palka P, et al. Echocardiographic characterization of cardiomyopathy in Friedreich's ataxia with tissue Doppler echocardiographically derived myocardial velocity gradients. Circulation 2000; 102:1276-82.

10 Palka $\mathbf{P}$, Lange A, Fleming RM, et al. Differences in myocardial velocity gradient measured throughout the cardiac cycle in patients with hypertrophic cardiomyopathy, athletes and patients with left ventricular hypertrophy due to hypertension. J Am Coll Cardiol 1997;30:760-8.

11 Nagueh SF, Bachinski LL, Meyer D, et al. Tissue Doppler imaging consistently detects myocardial abnormalities in patients with hypertrophic cardiomyopathy and provides a novel means for an early diagnosis before and independently of hypertrophy. Circulation 2001;104:128-30.

12 Dandel M, Hummel M, Muller J, et al. Reliability of tissue Doppler wall motion monitoring after heart transplantation for replacement of invasive routine screenings by optimally timed cardiac biopsies and catheterizations. Circulation 2001; 104(suppl 1):184-91.

13 Vinereanu D, lonescu AA, Fraser AG. Assessment of left ventricular long axis contraction can detect early myocardial dysfunction in asymptomatic patients with severe aortic regurgitation. Heart 2001;85:30-6.

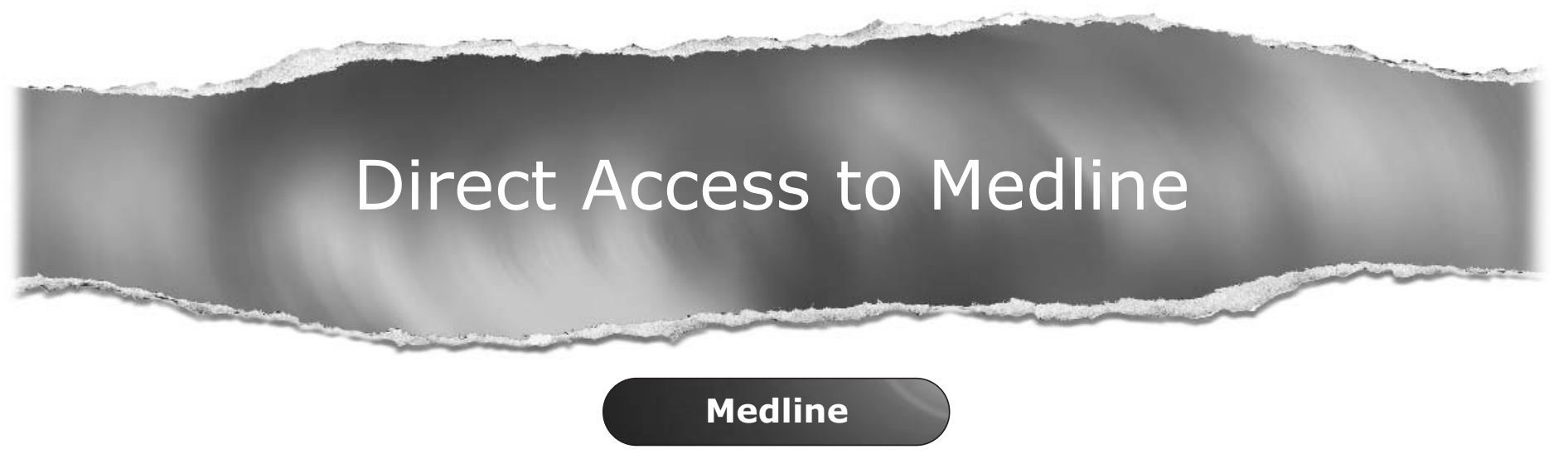

Link to Medline from the homepage and get straight into the National Library of Medicine's premier bibliographic database. Medline allows you to search across 9 million records of bibliographic citations and author abstracts from approximately 3,900 current biomedical journals. 